\section{Lupus anticoagulant is the main predictor of adverse pregnancy outcomes in aPL-positive patients: validation of PROMISSE study results}

Cecile M Yelnik, ${ }^{1,2}$ Carl A Laskin, ${ }^{3}$ T Flint Porter, ${ }^{4}$ D Ware Branch, ${ }^{4}$ Jill P Buyon, ${ }^{5}$ Marta M Guerra, ${ }^{1}$ Michael D Lockshin, ${ }^{1}$ Michelle Petri, ${ }^{6}$ Joan T Merrill, ${ }^{7}$ Lisa R Sammaritano, ${ }^{1}$ Mimi Y Kim, ${ }^{8}$ Jane E Salmon ${ }^{1}$

To cite: Yelnik CM, Laskin CA, Porter TF, et al. Lupus anticoagulant is the main predictor of adverse pregnancy outcomes in aPLpositive patients: validation of PROMISSE study results. Lupus Science \& Medicine 2016;3:e000131. doi:10.1136/lupus-2015000131

Received 2 October 2015 Revised 17 November 2015 Accepted 23 November 2015

CrossMark

For numbered affiliations see end of article.

Correspondence to Professor Jane E Salmon; salmonj@hss.edu

\section{ABSTRACT}

Objective: We previously reported that lupus anticoagulant (LAC) is the main predictor of poor pregnancy outcome in antiphospholipid antibody (aPL)-positive patients. We sought to confirm this finding in an independent group of patients who were subsequently recruited into the PROMISSE study. Methods: The PROMISSE study is a multicentre, prospective, observational study of pregnancy outcomes in women with aPL and/or systemic lupus erythematosus (SLE) that enrolled patients from 2003 to 2015. All consecutive, aPL-positive patients from the PROMISSE study who completed their pregnancy between April 2011 and January 2015 (after the previous PROMISSE report) are included in the current report. Patients were followed monthly until delivery, and aPL was tested at first, second and third trimesters of pregnancy and at 12 weeks post partum. Adverse pregnancy outcomes (APOs) were defined as fetal death after 12 weeks of gestation, neonatal death, delivery prior to 36 weeks of gestation due to preeclampsia or placental insufficiency or small-forgestational age (birth weight $<5$ th percentile).

Results: Forty-four aPL-positive patients are included in this paper. Thirteen patients had APOs, which occurred in $80 \%$ of cases during the second trimester of pregnancy. LAC was present in $69 \%$ of patients with APOs compared with $27 \%$ of patients without APOs $(p=0.01)$. No association was found between anticardiolipin antibodies ( $\mathrm{aCL}$ ) or anti- $\beta 2$ glycoprotein I antibodies (aß2GPI) IgG or IgM positivity and APOs. Definite antiphospholipid syndrome (history of thrombosis and/or pregnancy morbidity and $\mathrm{aPL}$ ) was found in $92 \%$ of patients with any APOs compared with $45 \%$ of patients without APOs ( $\mathrm{p}=0.004)$.

Conversely, the frequency of SLE was not statistically different between those with and without APOs (30\% vs $39 \%$ ).

Conclusions: Our findings, in an independent group of aPL-positive patients from the PROMISSE study, confirm that LAC, but not aCL and aß2GPI, is predictive of poor pregnancy outcomes after 12 weeks of pregnancy.

Trial registration number: NCT00198068.

\section{INTRODUCTION}

Lupus anticoagulant (LAC), anticardiolipin antibodies (aCL) and anti- $\beta 2$ glycoprotein I antibodies (aß2GPI) are associated with thrombosis and are included in the international classification criteria for antiphospholipid syndrome (APS). ${ }^{1}$ These antiphospholipid antibodies (aPL) are also associated with obstetrical morbidity, such as recurrent early miscarriage, otherwise unexplained fetal death and preterm delivery for pre-eclampsia or placental insufficiency. ${ }^{2}$ However, the association between specific aPL and pregnancy complications has not been precisely defined, and results from published studies have varied. ${ }^{3}$ Also, the prognostic value of the three accepted aPL in the prediction of pregnancy outcome is not well established.

The PROMISSE study (Predictors of pRegnancy Outcome: bioMarkers In antiphospholipid antibody Syndrome and Systemic lupus Erythematosus), a prospective multicentre observational study of pregnancies in women with systemic lupus erythematosus (SLE) and/or aPL, was designed to identify clinical features, laboratory tests and biomarkers that could be used to predict adverse pregnancy outcomes (APOs) after the first trimester of pregnancy. The initial analysis of data from PROMISSE, published in 2012, reported that LAC, but not aCL or aß2GPI, was a strong independent predictor of APO in aPL-positive patients. ${ }^{4}$ In this study, we aimed to validate the predictive value of different aPLs in an independent group of patients enrolled subsequent to those reported in our previous paper.

\section{PATIENTS AND METHODS}

Study population

All consecutive PROMISSE patients with aPL who finished their pregnancies between 
April 2011 and January 2015 are included; the previous study reported aPL-positive patients who delivered between September 2003 and March 2011. ${ }^{4}$ Inclusion and exclusion criteria are described elsewhere. ${ }^{4} 5$ Briefly, consecutive patients, aged 18-45 years, with singleton intrauterine pregnancy were enrolled before 18 weeks of gestation at six sites in North America and one in the UK.

\section{Data collection and follow-up}

The screening visit included medical history, physical examination and laboratory tests, including aPL. Patients were followed monthly during the pregnancy. Laboratory tests were repeated during the second (2023 weeks of gestation) and third trimesters (32-35 weeks of gestation) of pregnancy and at 3 months post partum. The patients' physicians made all treatment decisions.

\section{aPL assays}

aPL assays were performed in core laboratories as previously described and following the international guidelines for APS laboratory criteria. ${ }^{4}{ }^{5}$ For LAC determination, three screening tests (dilute Russell's viper venom time (dRVVT), dilute prothrombin time (dPT) and LAC-sensitive test for activated partial thromboplastin time (aPTT)) with confirmation were performed. Our previous report ${ }^{4}$ included patients with low aPL titre, defined as being negative for LAC and having aCL or a $32 \mathrm{GPI}$ titres $<40 \mathrm{IU} / \mathrm{mL}$. In the present study, only the presence of aCL and/or aß2GPI titres IgG or IgM $\geq 40$ GPL or MPL units, respectively, and/or LAC was considered positive, in accordance with Sapporo criteria. ${ }^{1}$

\section{Adverse pregnancy outcomes assessment}

APOs were determined as reported by the patients' obstetrician and included in the medical record. In equivocal cases, obstetrical members of the PROMISSE team adjudicated causes of fetal demise. APOs in PROMISSE were defined as: fetal death after 12 weeks of gestation, neonatal death before hospital discharge due to complications of prematurity, preterm delivery before 36 weeks of gestation due to gestational hypertension, pre-eclampsia or placental insufficiency and small-forgestational-age (SGA) neonate (birth weight, fifth percentile). ${ }^{4}{ }^{5}$ Other aetiologies for APOs were not included in the analyses.

\section{Statistical analysis}

Data are expressed as number (percentage), mean (SD) or median (InterQuartile). The association of patient characteristics with APOs was evaluated using the Fisher's exact or Mann-Whitney tests. A two-tailed $\mathrm{p}<0.05$ was considered statistically significant. Data were analysed using SPSS software package V.22.
RESULTS

\section{Baseline characteristics of the study population}

Forty-four new aPL-positive patients were enrolled in this study between April 2011 and January 2015 (table 1).

Thirteen patients had any APOs (table 2), which occurred primarily during the second trimester of pregnancy.

\section{Analysis of clinical and demographic predictors of APOs}

Age, race and ethnicity were similar in patients with and without APOs. Patients with APOs had higher body mass index (BMI) than patients without APOs $(p=0.04)$. Clinical APS and history of thrombosis were significantly associated with APOs $(p=0.004)$, whereas the diagnosis of SLE was not associated with APOs (table 1). The majority of the patients were treated with aspirin and/or low molecular weight heparin $(n=42)$. Given that few patients were untreated, no differences in pregnancy outcomes were evident in patients receiving these therapies. All patients who had APOs received aspirin and/ or heparin. Only two patients in this study did not receive antithrombotic prophylaxis; both were LAC negative and had successful pregnancies. The frequency of treatment with hydroxychloroquine was similar in those with and without APOs.

\section{Analysis of laboratory variables predictive of APO}

Among LAC-positive patients, 53\% (9/17) had APOs, including $29 \%$ with fetal death, while in LAC-negative patients $17 \%$ (4/24) had APOs with no fetal deaths. LAC was present at screening in $69 \%$ of the APO group compared with $27 \%$ of the non-APOs group ( $p=0.01$; table 1). Description of APOs according to the presence of LAC is shown in table 3.

The subgroup of patients with both prior thrombosis and LAC positivity were at very high risk of APOs (occurring in $78 \%$ in those patients). There was no difference in the frequency of aCL IgG between patients with and without APOs $(69 \%$ vs $55 \%$, respectively, $\mathrm{p}=0.37)$. Moreover, LAC was also present in six of nine patients who were aCL IgG positive and had APOs. A similar result was found for aß2GPI IgG $(61 \%$ vs $50 \%$, p=0.48), and LAC was present in five of eight patients who were aß2GPI positive and had APOs. APOs occurred in five of the six patients who were positive for all three aPL tests. Of laboratory tests measured during the second trimester, when the majority of APOs occurred, LAC remained the only aPL associated with APOs.

Of note, five patients had pregnancy complications (two HELLP pre-eclampsia after 36 weeks of gestation and three SGA $<10$ th percentile) that did not fulfil the PROMISSE study definition of APOs. Among those patients, four of five were LAC positive. When the definition of APOs is expanded to include these five patients yielding a total of $18 \mathrm{APO}$, the rate of APOs was significantly higher in LAC-positive patients compared with LAC-negative patients $(76 \%$ vs $21 \%, \mathrm{p}=0.01)$. The presence of other aPLs remained not significant. 
Table 1 Baseline characteristics of the study population

\begin{tabular}{|c|c|c|c|c|}
\hline Characteristics & $\begin{array}{l}\text { Total population } \\
(n=44)\end{array}$ & $\begin{array}{l}\text { APOs patients } \\
(n=13)\end{array}$ & $\begin{array}{l}\text { No APOs patients } \\
(n=31)\end{array}$ & p Value* \\
\hline Age (years) $\dagger$ & $32.3(4.6)$ & $32.2(5.6)$ & $32.3(4.2)$ & 0.69 \\
\hline \multicolumn{5}{|l|}{ Ethnicity } \\
\hline Hispanic & $4(9 \%)$ & $2(15 \%)$ & $2(6 \%)$ & \multirow{2}{*}{0.67} \\
\hline Non-Hispanic & $40(91 \%)$ & $11(85 \%)$ & $29(93 \%)$ & \\
\hline \multicolumn{5}{|l|}{ Race } \\
\hline White & $36(82 \%)$ & $8(73 \%)$ & $28(93.3 \%)$ & \multirow[t]{2}{*}{0.11} \\
\hline Non-white & $5(11 \%)$ & $3(23 \%)$ & $2(6 \%)$ & \\
\hline \multicolumn{5}{|l|}{ BMI‡ } \\
\hline$<25$ & $28(64 \%)$ & $5(38 \%)$ & $23(74 \%)$ & \multirow[t]{3}{*}{0.04} \\
\hline $25-30$ & $10(23 \%)$ & $6(46 \%)$ & $4(13 \%)$ & \\
\hline$>30$ & $6(14 \%)$ & $2(15 \%)$ & $4(13 \%)$ & \\
\hline SLE & $16(36 \%)$ & $4(30 \%)$ & $12(39 \%)$ & 0.74 \\
\hline Number previous pregnancies & $1.7(1.6)$ & $1.6(1.6)$ & $1.7(1.7)$ & 0.80 \\
\hline APS & $26(59 \%)$ & $12(92 \%)$ & $14(45 \%)$ & 0.004 \\
\hline History of thrombosis & $13(29 \%)$ & $9(69 \%)$ & $4(13 \%)$ & 0.0001 \\
\hline History of obstetrical complications & $18(41 \%)$ & $8(61 \%)$ & $10(31 \%)$ & 0.07 \\
\hline \multicolumn{5}{|l|}{ Treatment during pregnancy } \\
\hline Hydroxychloroquine & 17 (39\%) & $6(46 \%)$ & $11(35 \%)$ & 0.44 \\
\hline Corticosteroid & $4(9 \%)$ & $1(8 \%)$ & $3(10 \%)$ & 1.0 \\
\hline Aspirin & $36(82 \%)$ & $10(77 \%)$ & $26(84 \%)$ & 0.67 \\
\hline Heparin & $32(73 \%)$ & $12(92 \%)$ & $20(64 \%)$ & 0.07 \\
\hline Heparin and/or aspirin & $42(95 \%)$ & $13(100 \%)$ & $29(94 \%)$ & 0.37 \\
\hline Azathioprine & $1(2 \%)$ & $0(0 \%)$ & $1(3.2 \%)$ & \\
\hline IVIG once per month & $1(2 \%)$ & $1(8 \%)$ & $0(0 \%)$ & \\
\hline \multicolumn{5}{|l|}{ aPL positive at screening§ } \\
\hline LAC & 17 (39\%) & $9(69 \%)$ & $8(27 \%)$ & 0.01 \\
\hline $\mathrm{aCL} \lg \mathrm{G}$ & $26(59 \%)$ & $9(69 \%)$ & $17(55 \%)$ & 0.37 \\
\hline aß2GPI IgG & $23(52 \%)$ & $8(61 \%)$ & $15(50 \%)$ & 0.48 \\
\hline aß2GPI and/or aCL IgM & $8(9 \%)$ & $1(8 \%)$ & $7(23 \%)$ & 0.4 \\
\hline Triple aPL positivity & $6(14 \%)$ & $5(38 \%)$ & $1(3 \%)$ & 0.008 \\
\hline
\end{tabular}

*Fisher's exact or Mann-Whitney tests compared patients with APOs to patients without APOs.

†Age was expressed as mean (SD). Other characteristics were expressed as number (\% of the total).

$\ddagger \mathrm{BMI}$ comparison was done between groups $<25$ vs $\geq 25$.

$\S A P L$ positivity for each test was defined as lupus anticoagulant: RVVT, dilute TTI or PTT LA with confirmation; aCL: IgG $\geq 40$ GPL units; IgM $\geq 40$ MPL units; and anti-32GPI: IgG $\geq 40$ GPL units; IgM $\geq 40$ MPL units. To be considered positive, each test met these criteria at least twice between 6 weeks and 5 years apart of which one must be during the PROMISSE pregnancy at a core lab. ${ }^{4}$ Triple aPL positivity was defined as having all three aPL tests positive.

aCL, anticardiolipin antibodies; aPL, antiphospholipid antibodies; APOs, adverse pregnancy outcomes; APS, antiphospholipid syndrome; aß2GPI, anti- $\beta 2$ glycoprotein I antibodies; BMI, body mass index; IU, international unit; IVIG, intravenous immunoglobulin therapy; LAC, lupus anticoagulant; SLE, systemic lupus erythematosus.

\section{DISCUSSION}

In this report, we show that LAC was the only aPL associated with APOs after the first trimester, confirming the findings from our previous study in an independent group of aPL-positive patients. ${ }^{4}$ We also confirmed that clinical APS and history of thrombosis are strong risk factors for APOs. ${ }^{4}$

Despite the small sample size, the present study has strengths, including prospective design and multicentre recruitment of a precisely characterised, homogeneous study population with regard to aPL determinations. All aPL assays were performed in core laboratories and international classification criteria were strictly followed. We determined LAC to be present if any of three screening tests (dRVVT, dPT or aPTT) followed by confirming tests were abnormal, because any single positive test was not predictive of outcomes and no single test was superior. ${ }^{4}$ Hence, in clinical practice, excluding LAC with a single screening test may be insufficient, but this approach may be impractical. The main limitation was the inability to perform a multivariate analysis to adjust for potential confounders because of the small number of patients and APOs.

Our previous report described findings from a prospective cohort of 144 patients from PROMISSE. ${ }^{4}$ Differences in study design make comparison of our results with those published in other studies difficult. Consistent with our findings, Helgadottir et at ${ }^{6}$ reported that LAC, but not aß2GPI nor aCL, was the only aPL associated with a history of fetal death after 26 weeks of gestation in a retrospective study, which included 105 cases compared with 262 controls with live births. Data prospectively and retrospectively collected on 247 patients with obstetrical APS from the European Registry 
Table 2 Description of the 13 patients with adverse pregnancy outcomes

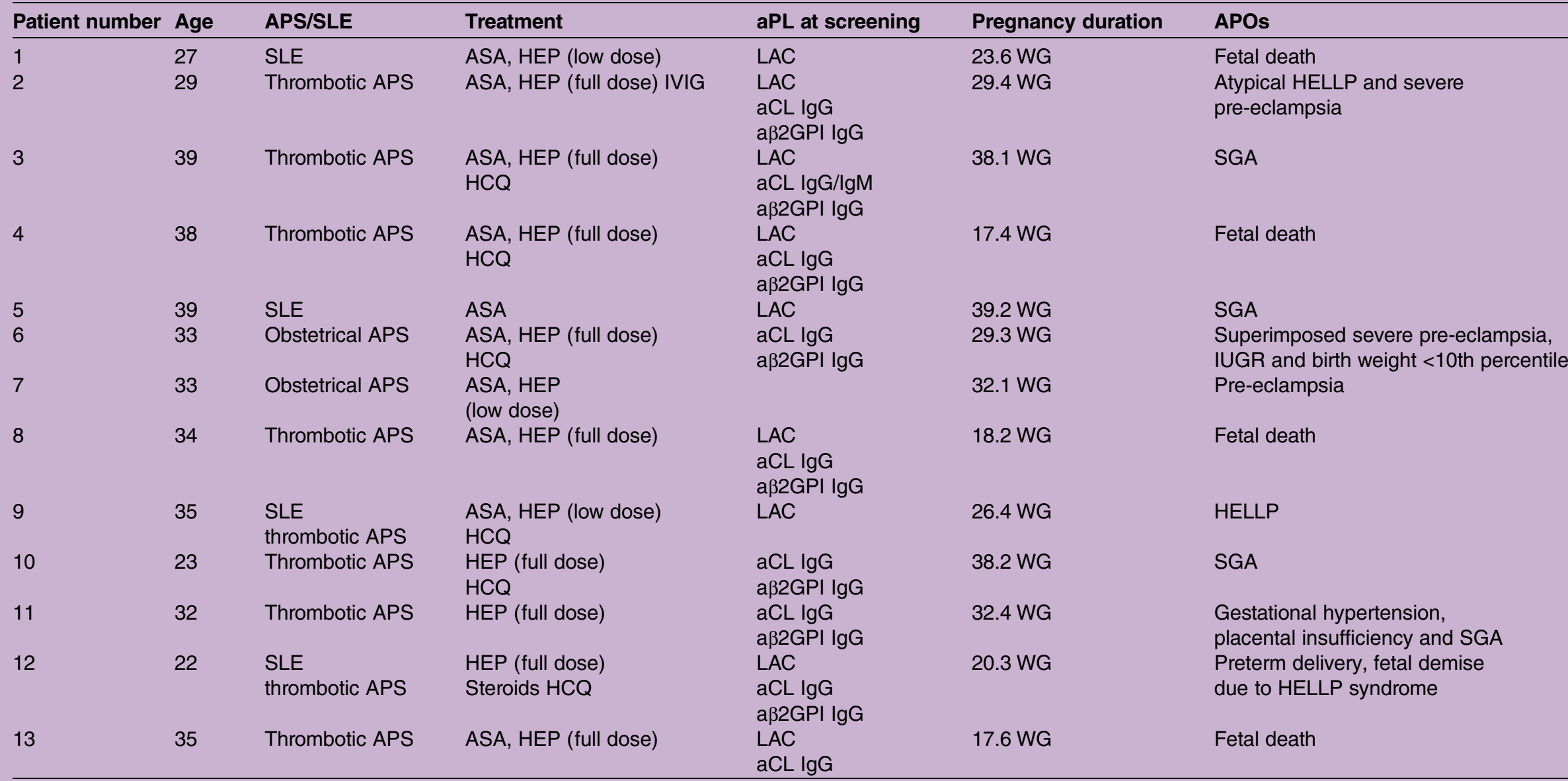

aCL, anticardiolipin antibodies; aPL, antiphospholipid antibodies; APOs, adverse pregnancy outcomes; APS, antiphospholipid syndrome; ASA, aspirin; aß2GPI, anti-32 glycoprotein I antibodies; HCQ, hydroxychloroquinine; HELLP, haemolysis elevated liver enzyme and low platelet count syndrome; HEP, low molecular weight heparin; IUGR, intrauterine growth restriction; IVIG,

intravenous immunoglobulin therapy; LAC, lupus anticoagulant; SGA, small for gestational age neonate (birth weight below the fifth percentile); SLE, systemic lupus erythematosus;

WG, weeks of gestation. 
Table 3 Adverse pregnancy outcomes among LAC-positive and LAC-negative patients

\begin{tabular}{lllll}
\hline Patients & APOs & Fetal death & $\begin{array}{l}\text { Preterm delivery } \\
\text { due to placental causes }\end{array}$ & SGA <5th percentile \\
\hline LAC positive $(n=17)$ & $9(53 \%)$ & $5(29 \%)$ & $3(18 \%)$ & $2(12 \%)$ \\
LAC negative $(n=24)$ & $4(17 \%)$ & $0(0 \%)$ & $1(4 \%)$ & $2(8 \%)$ \\
\hline
\end{tabular}

Preterm delivery due to placental-related diseases: delivery before 36 weeks of gestation due to gestational hypertension, pre-eclampsia or placental insufficiency. Data expressed as number (\% of the line).

APOs, adverse pregnancy outcomes; LAC, lupus anticoagulant; SGA, small for gestational-age neonate (birth weight below the fifth percentile).

of APS showed that LAC and triple aPL positivity, but not single aPL positivity, was associated with early and late obstetrical complications. ${ }^{7}$ Others have argued that LAC alone is not as significant a risk factor as is the presence of triple aPL positivity suggested to be the independent predictor of poor pregnancy outcomes. ${ }^{8}{ }^{9}$ In contrast, our findings show that in two separate prospective cohorts, LAC positivity was sufficient to predict risk of APOs regardless of the association with aCL or aß2GPI positivity.

Because medications were at the discretion of the treating physician, our study was not designed to evaluate treatment. Nonetheless, both of the current and our previous report did not show a beneficial effect of low molecular weight heparin. This result may reflect the bias of physicians to treat patients they consider at higher risk for APOs more intensely, or that low molecular weight heparin is not effective. ${ }^{10} 11$ Others reported, in a meta-analysis, no preventive effect of heparin against obstetrical complications. Of note, only asymptomatic aPL carriers (patients without any history of thrombosis or obstetrical morbidity) were included in this meta-analysis, whereas in our study only $40 \%$ were asymptomatic carriers. ${ }^{12}$

\section{CONCLUSION}

This study independently confirmed that LAC is the only aPL predictor of poor pregnancy outcomes after the first trimester of pregnancy in aPL-positive patients.

\footnotetext{
Author affiliations

${ }^{1}$ Department of Medicine, Hospital for Special Surgery, New York City, New York, USA

${ }^{2}$ Internal Medicine Department, University of Lille, UFR Médecine, Lille, France

${ }^{3}$ University of Toronto and LifeQuest Centre for Reproductive Medicine,

Toronto, Canada

${ }^{4}$ Department of Obstetrics and Gynecology, University of Utah and Intermountain Healthcare, Salt Lake City, Utah, USA

${ }^{5}$ Department of Medicine, New York University School of Medicine, New York City, New York, USA

${ }^{6}$ Department of Medicine, Johns Hopkins University School of Medicine, Baltimore, Maryland, USA

${ }^{7}$ Department of Medicine, Oklahoma Medical Research Foundation, Oklahoma City, Oklahoma, USA

${ }^{8}$ Department of Epidemiology and Public Health, Albert Einstein College of Medicine, New York City, New York, USA
}

Funding National Institute of Arthritis and Musculoskeletal and Skin Diseases of the National Institutes of Health AR49772.

\section{Competing interests None declared.}

Ethics approval IRBs of the different institutions approved the study.

Provenance and peer review Not commissioned; externally peer reviewed.

Data sharing statement Because the investigative team is still analysing the data for biomarkers and genetics, at this time we are not willing to make the protocol or data public except for what is currently published in clinicaltrials. gov.

Open Access This is an Open Access article distributed in accordance with the Creative Commons Attribution Non Commercial (CC BY-NC 4.0) license, which permits others to distribute, remix, adapt, build upon this work noncommercially, and license their derivative works on different terms, provided the original work is properly cited and the use is non-commercial. See: http:// creativecommons.org/licenses/by-nc/4.0/

\section{REFERENCES}

1. Miyakis S, Lockshin MD, Atsumi T, et al. International consensus statement on an update of the classification criteria for definite antiphospholipid syndrome (APS). J Thromb Haemost 2006;4:295-306.

2. Abou-Nassar K, Carrier M, Ramsay T, et al. The association between antiphospholipid antibodies and placenta mediated complications: a systematic review and meta-analysis. Thromb Res 2011;128:77-85.

3. De Jesus GR, Agmon-Levin N, Andrade CA, et al. 14th International Congress on Antiphospholipid Antibodies Task Force report on obstetric antiphospholipid syndrome. Autoimmun Rev 2014;13:795-813.

4. Lockshin MD, Kim M, Laskin CA, et al. Prediction of adverse pregnancy outcome by the presence of lupus anticoagulant, but not anticardiolipin antibody, in patients with antiphospholipid antibodies. Arthritis Rheum 2012;64:2311-8.

5. Buyon JP, Kim MY, Guerra MM, et al. Predictors of pregnancy outcomes in patients with lupus: a cohort study. Ann Intern Med 2015;163:153-63.

6. Helgadottir LB, Skjeldestad FE, Jacobsen AF, et al. The association of antiphospholipid antibodies with intrauterine fetal death: a case-control study. Thromb Res 2012;130:32-7.

7. Alijotas-Reig J, Ferrer-Oliveras R, Ruffatti A, et al. The European Registry on Obstetric Antiphospholipid Syndrome (EUROAPS): a survey of 247 consecutive cases. Autoimmun Rev 2015;14:387-95.

8. Ruffatti A, Calligaro A, Hoxha A, et al. Laboratory and clinical features of pregnant women with antiphospholipid syndrome and neonatal outcome. Arthritis Care Res 2010;62:302-7.

9. Ruffatti A, Tonello M, Visentin MS, et al. Risk factors for pregnancy failure in patients with anti-phospholipid syndrome treated with conventional therapies: a multicentre, case-control study. Rheumatol Oxf Engl 2011;50:1684-9.

10. Ruiz-Irastorza G, Crowther M, Branch W, et al. Antiphospholipid syndrome. Lancet 2010;376:1498-509.

11. Mak A, Cheung MW-L, Cheak AA, et al. Combination of heparin and aspirin is superior to aspirin alone in enhancing live births in patients with recurrent pregnancy loss and positive anti-phospholipid antibodies: a meta-analysis of randomized controlled trials and meta-regression. Rheumatol Oxf Engl 2010;49:281-8.

12. Amengual O, Fujita D, Ota E, et al. Primary prophylaxis to prevent obstetric complications in asymptomatic women with antiphospholipid antibodies: a systematic review. Lupus 2015;24:1135-42. 